\title{
Crystal structure of 2,2'-bis(4,5-dimethylimidazole)(dithiocyanato)mercury(II), $\mathrm{Hg}\left(\mathrm{C}_{10} \mathrm{H}_{14} \mathrm{~N}_{4}\right)(\mathrm{CNS})_{2}$
}

\author{
A. R. Mahjoub, ${ }^{*,}$, A. Ramazani ${ }^{\mathrm{II}}$ and A. Morsali ${ }^{\mathrm{I}}$ \\ I Tarbiat Modarres University, School of Science, Department of Chemistry, P. O. Box 14155-4838, Tehran, I. R. Iran \\ II University of Zanjan, Department of Chemistry, P. O. Box 45195-313, Zanjan, I. R. Iran
}

Received June 2, 2003, accepted in revised form and available on-line October 28, 2003; CCDC-No. 1267/1100

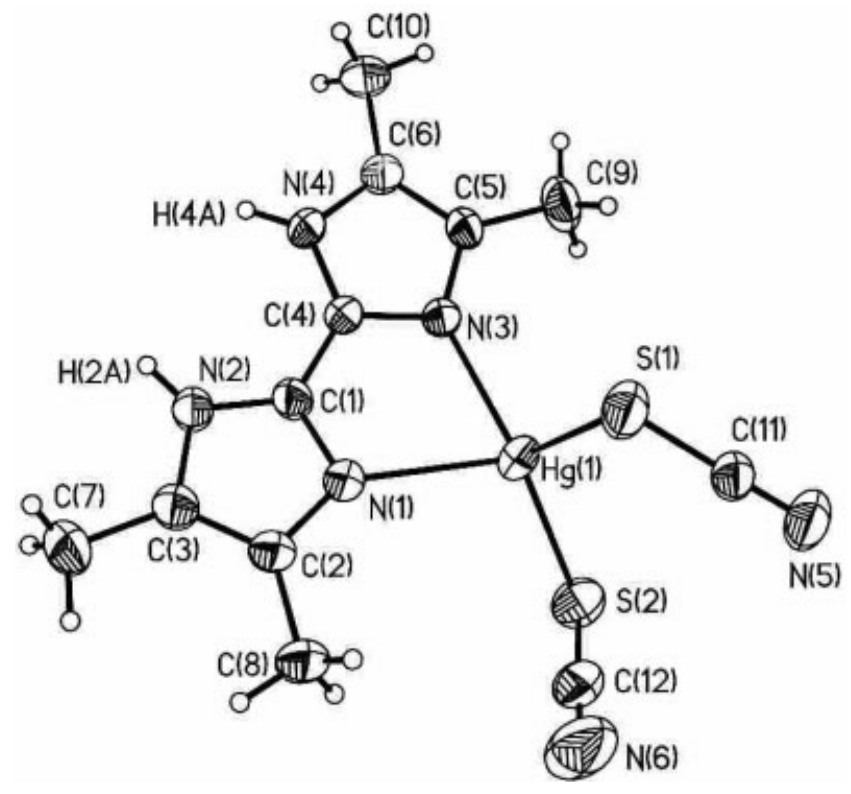

Abstract

$\mathrm{C}_{12} \mathrm{H}_{14} \mathrm{HgN}_{6} \mathrm{~S}_{2}$, triclinic, $P \overline{1}$ (No. 2), $a=7.732(2) \AA$,

$b=10.252(2) \AA, c=11.802(2) \AA, \alpha=65.57(3)^{\circ}, \beta=72.19(3)^{\circ}$,

$\gamma=88.13(3)^{\circ}, V=806.2 \AA^{3}, Z=2, R_{\mathrm{gt}}(F)=0.048$,

$w R_{\mathrm{ref}}\left(F^{2}\right)=0.123, T=293 \mathrm{~K}$.

\section{Source of material}

The complex was prepared by dissolving mercury(II) thiocyanate $(0.316 \mathrm{~g}, 1 \mathrm{mmol})$ in distillation water and adding an alcoholic solution of 2,2'-bis(4,5-dimetyleimidazole) ligand (0.190 g, $2 \mathrm{mmol})$. The resulting solution was stirred for $5 \mathrm{~h}$ at room temperature, then it was allowed to stand for 2-3 days in a refrigerator (ca. $6{ }^{\circ} \mathrm{C}$ ). Black crystals of the desired product precipitated, which were filtered off, washed with acetone and ether and air dried $(0.354 \mathrm{~g}$, yield $70 \%)$. Analysis: found - C, $28.61 \%$; H, 2.68\%; N, $16.43 \%$; calculated for $\mathrm{C}_{12} \mathrm{H}_{14} \mathrm{HgN}_{6} \mathrm{~S}_{2}-\mathrm{C}, 28.40 \% ; \mathrm{H}, 2.76 \%$; $16.60 \%$. Melting points were measured on an Electrothermal 9100 apparatus and are uncorrected. Elemental analyses were performed using a Heraeus CHN-O-Rapid analyzer.

\section{Discussion}

The ability of mercury(II) salts to form a wide variety of 1:1 and 1:2 complexes, with neutral ligands has been known for some time [1]. Most of these complexes contain halide ions and the thiocyanate ion have been rarely reported [2]. This ion is an ambidentate ligand and can coordinate via both $\mathrm{N}$ and $\mathrm{S}$ atoms.

\footnotetext{
* Correspondence author (e-mail: mahjouba@net1cs.modares.ac.ir)
}

The coordination mode depends on the nature of the metal center. Hence $\mathrm{N}$-donor atoms are found in $\mathrm{Zn}$ complexes, while in mercury(II) complexes, the $\mathrm{S}$ atom is undoubtedly the expected ligating site for $\mathrm{Hg}^{+2}$ [2]. The complex is built up of a monomeric $\mathrm{Hg}(\mathrm{SCN})_{2}$ unit $[d(\mathrm{Hg} 1-\mathrm{S} 1)=2.539(2) \AA$ and $d(\mathrm{Hg} 1-\mathrm{S} 2)=$ $2.408(2) \AA$ ] , with one 2,2' -bis(4,5-dimetylimidazole) ligand coordinating the $\mathrm{Hg}$ atom via the two $\mathrm{N}$ atoms. This gives rise to a five-member chelate ring $\mathrm{Hg} / \mathrm{N} 1 / \mathrm{C} 1 / \mathrm{C} 4 / \mathrm{N} 3: d(\mathrm{Hg} 1-\mathrm{N} 1)=$ $2.250(5) \AA$ and $d(\operatorname{Hg} 1-\mathrm{N} 3)=2.403(6) \AA$, and to a distorted tetrahedral environment for $\mathrm{Hg}$ atoms. The smallest and largest bond angles around the $\mathrm{Hg}$ atoms are $\angle \mathrm{N} 3-\mathrm{Hg} 1-\mathrm{S} 2=140.5(2)^{\circ}$ and $\angle \mathrm{N} 1-\mathrm{Hg} 1-\mathrm{N} 3=74.2(2)^{\circ}$, respectively. There is $\pi-\pi$ stacking interaction (charge-transfer arrays) [2] between the parallel aromatic rings belonging to adjacent chains. In the planar species the mean molecular planes are close to parallel and separated by a distance of $\sim 3.5 \AA$, which is close to that of the planes in graphite. The complexes are linked by hydrogen bonds. The coordinating 2,2'-bis(4,5-dimetylimidazole) molecule is involved in hydrogen bonding acting as hydrogen-bond donor with $\mathrm{N}$ atoms of thiocyanate ions as potential hydrogen-bond acceptors. Each complex is bonded to two neighbors. Both amine $\mathrm{H}$ atoms are hydrogen bonded to $\mathrm{N}$ atoms.

Table 1. Data collection and handling.

Crystal:

Wavelength:

$\mu$ :

Diffractometer, scan mode: $2 \theta_{\max }$ :

$N(h k l)_{\text {measured }}, N(h k l)_{\text {unique }}$ Criterion for $I_{\mathrm{obs}}, N(\mathrm{hkl})_{\mathrm{gt}}$ : $N$ (param) refined:

Programs:

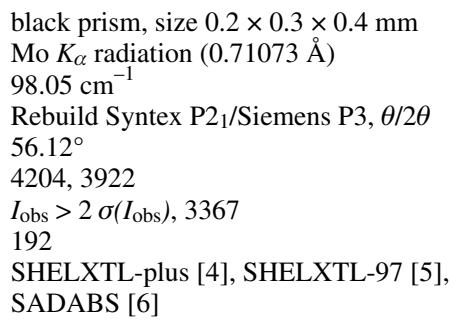

black prism, size $0.2 \times 0.3 \times 0.4 \mathrm{~mm}$ Mo $K_{\alpha}$ radiation $(0.71073 \AA$ ) $98.05 \mathrm{~cm}^{-1}$

Rebuild Syntex P2 1 /Siemens P3, $\theta / 2 \theta$ $56.12^{\circ}$

4204, 3922

$I_{\mathrm{obs}}>2 \sigma\left(I_{\mathrm{obs}}\right), 3367$

192

SHELXTL-plus [4], SHELXTL-97 [5], SADABS [6]

Table 2. Atomic coordinates and displacement parameters (in $\AA^{2}$ ).

\begin{tabular}{lllrll}
\hline Atom & Site & $x$ & $y$ & $z$ & $U_{\text {iso }}$ \\
\hline $\mathrm{H}(11 \mathrm{~A})$ & $2 i$ & 0.1859 & -0.3725 & 0.5840 & 0.097 \\
$\mathrm{H}(11 \mathrm{~B})$ & $2 i$ & 0.2765 & -0.4389 & 0.4846 & 0.097 \\
$\mathrm{H}(11 \mathrm{C})$ & $2 i$ & 0.0654 & -0.4287 & 0.5247 & 0.097 \\
$\mathrm{H}(10 \mathrm{~A})$ & $2 i$ & 0.2333 & -0.1416 & 0.5722 & 0.084 \\
$\mathrm{H}(10 \mathrm{~B})$ & $2 i$ & 0.1583 & 0.0041 & 0.5033 & 0.084 \\
$\mathrm{H}(10 \mathrm{C})$ & $2 i$ & 0.3693 & -0.0067 & 0.4645 & 0.084 \\
$\mathrm{H}(12 \mathrm{~A})$ & $2 i$ & 0.3213 & 0.3226 & -0.3151 & 0.079 \\
$\mathrm{H}(12 \mathrm{~B})$ & $2 i$ & 0.4504 & 0.3619 & -0.2515 & 0.079 \\
& & & & &
\end{tabular}


Table 2. Continued.

\begin{tabular}{lllrrr}
\hline Atom & Site & $x$ & $y$ & $z$ & $U_{\text {iso }}$ \\
\hline $\mathrm{H}(12 \mathrm{C})$ & $2 i$ & 0.2412 & 0.3784 & -0.2064 & 0.079 \\
$\mathrm{H}(13 \mathrm{~A})$ & $2 i$ & 0.2622 & 0.1125 & -0.3296 & 0.075 \\
$\mathrm{H}(13 \mathrm{~B})$ & $2 i$ & 0.1202 & -0.0220 & -0.2300 & 0.075 \\
$\mathrm{H}(13 \mathrm{C})$ & $2 i$ & 0.3291 & -0.0401 & -0.2726 & 0.075 \\
$\mathrm{H}(2 \mathrm{~A})$ & $2 i$ & 0.2005 & -0.2866 & 0.2494 & 0.040 \\
$\mathrm{H}(4 \mathrm{~A})$ & $2 i$ & 0.2091 & -0.1528 & 0.0028 & 0.030 \\
\hline
\end{tabular}

Table 3. Atomic coordinates and displacement parameters (in $\AA^{2}$ ).

\begin{tabular}{|c|c|c|c|c|c|c|c|c|c|c|}
\hline Atom & Site & $x$ & $y$ & $z$ & $U_{11}$ & $U_{22}$ & $U_{33}$ & $U_{12}$ & $U_{13}$ & $U_{23}$ \\
\hline $\mathrm{Hg}(1)$ & $2 i$ & $0.32749(4)$ & $0.24935(3)$ & $0.11568(3)$ & $0.0629(2)$ & $0.0337(2)$ & $0.0564(2)$ & $0.0089(1)$ & $-0.0306(1)$ & $-0.0260(1)$ \\
\hline$S(1)$ & $2 i$ & $0.0266(3)$ & $0.3568(2)$ & $0.1570(2)$ & $0.0476(8)$ & $0.0367(9)$ & $0.070(1)$ & $0.0044(7)$ & $-0.0221(8)$ & $-0.0245(8)$ \\
\hline$S(2)$ & $2 i$ & $0.5583(3)$ & $0.3612(2)$ & $0.1551(2)$ & $0.0517(9)$ & $0.053(1)$ & $0.064(1)$ & $-0.0034(8)$ & $-0.0237(9)$ & $-0.026(1)$ \\
\hline $\mathrm{N}(1)$ & $2 i$ & $0.2617(8)$ & $-0.0029(6)$ & $0.2612(5)$ & $0.056(3)$ & $0.029(3)$ & $0.035(3)$ & $0.003(2)$ & $-0.021(2)$ & $-0.012(2)$ \\
\hline $\mathrm{N}(2)$ & $2 i$ & $0.2153(9)$ & $-0.2185(6)$ & $0.2721(5)$ & $0.069(4)$ & $0.030(3)$ & $0.030(3)$ & $0.004(2)$ & $-0.018(3)$ & $-0.013(2)$ \\
\hline $\mathrm{N}(3)$ & $2 i$ & $0.2997(8)$ & $0.1375(6)$ & $-0.0076(5)$ & $0.052(3)$ & $0.028(2)$ & $0.029(2)$ & $0.007(2)$ & $-0.018(2)$ & $-0.014(2)$ \\
\hline $\mathrm{N}(5)$ & $2 i$ & $0.166(1)$ & $0.6196(8)$ & $0.1293(8)$ & $0.097(5)$ & $0.035(3)$ & $0.061(4)$ & $0.006(3)$ & $-0.028(4)$ & $-0.024(3)$ \\
\hline $\mathrm{N}(6)$ & $2 i$ & $0.358(2)$ & $0.328(1)$ & $0.411(1)$ & $0.19(1)$ & $0.080(7)$ & $0.055(5)$ & $-0.023(8)$ & $-0.031(6)$ & $-0.029(5)$ \\
\hline $\mathrm{C}(1)$ & $2 i$ & $0.2450(9)$ & $-0.0776(7)$ & $0.1962(6)$ & $0.052(3)$ & $0.030(3)$ & $0.027(3)$ & $0.008(2)$ & $-0.015(2)$ & $-0.013(2)$ \\
\hline $\mathrm{C}(2)$ & $2 i$ & $0.241(1)$ & $-0.1021(8)$ & $0.3875(6)$ & $0.055(4)$ & $0.038(3)$ & $0.031(3)$ & $0.001(3)$ & $-0.017(3)$ & $-0.014(3)$ \\
\hline $\mathrm{C}(3)$ & $2 i$ & $0.213(1)$ & $-0.2373(8)$ & $0.3963(7)$ & $0.064(4)$ & $0.037(3)$ & $0.032(3)$ & $0.005(3)$ & $-0.017(3)$ & $-0.015(3)$ \\
\hline $\mathrm{C}(4)$ & $2 i$ & $0.2607(9)$ & $-0.0054(7)$ & $0.0584(6)$ & $0.048(3)$ & $0.028(3)$ & $0.035(3)$ & $0.008(2)$ & $-0.020(3)$ & $-0.016(2)$ \\
\hline$C(5)$ & $2 i$ & $0.2956(9)$ & $0.1724(7)$ & $-0.1333(6)$ & $0.047(3)$ & $0.028(3)$ & $0.030(3)$ & $0.006(2)$ & $-0.016(3)$ & $-0.009(2)$ \\
\hline$C(6)$ & $2 i$ & $0.2590(9)$ & $0.0481(7)$ & $-0.1417(6)$ & $0.054(3)$ & $0.034(3)$ & $0.029(3)$ & $0.005(3)$ & $-0.017(3)$ & $-0.016(2)$ \\
\hline $\mathrm{C}(8)$ & $2 i$ & $0.251(1)$ & $-0.0576(9)$ & $0.4911(7)$ & $0.091(6)$ & $0.048(4)$ & $0.035(4)$ & $0.000(4)$ & $-0.026(4)$ & $-0.019(3)$ \\
\hline $\mathrm{C}(9)$ & $2 i$ & $0.330(1)$ & $0.3219(8)$ & $-0.2355(8)$ & $0.074(5)$ & $0.033(3)$ & $0.041(4)$ & $0.010(3)$ & $-0.019(3)$ & $-0.005(3)$ \\
\hline$C(10)$ & $2 i$ & $0.241(1)$ & $0.0223(9)$ & $-0.2535(7)$ & $0.071(4)$ & $0.052(4)$ & $0.039(4)$ & $0.008(4)$ & $-0.027(3)$ & $-0.025(3)$ \\
\hline $\mathrm{C}(11)$ & $2 i$ & $0.110(1)$ & $0.5109(7)$ & $0.1414(7)$ & $0.056(4)$ & $0.031(3)$ & $0.035(3)$ & $0.006(3)$ & $-0.016(3)$ & $-0.012(3)$ \\
\hline $\mathrm{C}(12)$ & $2 i$ & $0.434(1)$ & $0.3393(9)$ & $0.3075(9)$ & $0.083(6)$ & $0.043(4)$ & $0.060(5)$ & $-0.008(4)$ & $-0.036(4)$ & $-0.020(4)$ \\
\hline
\end{tabular}

Acknowledgments. Support of this investigation by Tarbiat Modarres University and Zanjan University is gratefully acknowledged. We thank the Institute of Organoelement Compounds of the Russian Academy of Science for determining the crystal structure by $\mathrm{X}$-ray diffraction methods.

\section{References}

1. Mahjoub, A. R.; Morsali, A.: Hg(II), Tl(III), Cu(I), and Pd(II) Complexes With 2,2'-diphenyl-4,4'-bithiazole (DPBTZ), Syntheses and X-ray Crystal structure of $\left.[\mathrm{Hg}(\mathrm{DPBTZ})(\mathrm{SCN}))_{2}\right]$. J. Coord. Chem. (2003), accepted.

2. Grenwood, N. N.; Earnshaw, A.: Chemistry of the Elements, p. 235-236, Pergamon Press, Oxford 1984.

3. Fengli, B.; Fangfang, J.; Yujie, Y.; Lude, L.; Xin, W.; Shanmuga, S. R.; Hoong-Kunm, F.: 1,4-Bis(2-benzimidazole)benzene. Acta Crystallogr. C56 (2000) 718-719.
4. Sheldrick G. M.: SHELXTL-plus, Version 5.o. Software Reference Map Siemens Industrial Automation, Inc., Madison, USA 1995.

5. Sheldrick, G. M.: SHELXTL-97. A Program for Refining Crystal Structures. Version 5.1o, Bruker AXS Inc., Madison, WI-53719, USA 1997. 13.

6. Sheldrick, G. M.: SADABS, Bruker AXS Inc., Madison, WI-53719, USA 1997. 